\title{
Psychological impact of receiving negative BRCA1 mutation test results in Ashkenazim
}

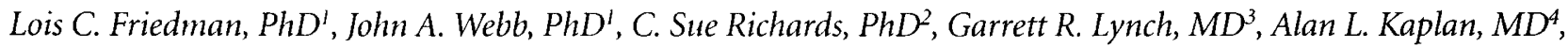
F. Charles Brunicardi, $M D^{5}$, and Sharon E. Plon, $M D, P h D^{2,6,7}$

\begin{abstract}
Purpose: Most DNA test results for breast/ovarian cancer susceptibility are negative. Because negative test results might be interpreted incorrectly and may have serious psychological and behavioral implications, determining the psychological impact of such results is important. Methods: A community-based sample of 289 Ashkenazim was tested for 185delAG. The 199 mutation-negatives provided data at baseline and follow-up. Increased risk participants included those who received negative test results but remained at increased risk because positive family and/or personal histories of breast or ovarian cancer made the results uninformative. Average risk meant those who tested negative and had negative family and personal histories of breast or ovarian cancer. Using a logistic regression analysis, both groups' psychological distress levels were compared at baseline and at 1 and 6 months after notification of DNA test results. Results: A logistic regression analysis showed significant but small differences in cancer-specific distress after 6 months between increased and average risk participants $(P<0.006)$. Increased risk participants reported more distress than average risk. General distress declined among all participants after 1 month. Although baseline and follow-up differences in cancer-specific distress obtained by the increased and average risk participants were statistically significant, none of the absolute levels observed reflected especially high degrees of stress. Conclusions: Receipt of negative DNA test results does not have a deleterious psychological impact, whether results are informative or uninformative. Genetics in Medicine, 1999;1(3):74-79
\end{abstract}

Key words: negative BRCA1 mutation results, Ashkenazim, community-based testing, psychological impact

\section{INTRODUCTION}

Approximately one in eight women in the United States eventually will develop breast cancer. ${ }^{1}$ Although most breast cancer is caused by somatic mutations, approximately $5 \%$ to $10 \%$ of all breast cancer is inherited as a result of a germ-line mutation in a breast cancer susceptibility gene. ${ }^{2,3}$ Among Ashkenazi Jews, three germ-line mutations have been identified. The $B R C A 1$ mutation, 185 delAG, is associated with both a high rate and an early onset of breast and ovarian cancer. ${ }^{4,5}$ The carrier frequency for this mutation has been reported to be approximately $1 \%$ in the Ashkenazi Jewish population. ${ }^{6}$ Another BRCA1 mutation, 5382 ins $C$, has been identified with a reported carrier frequency of $0.13 \%$ in Ashkenazim. ${ }^{6}$

After this study was underway, a second common Jewish mutation, 6174delT in the BRCA2 gene, was found to increase the risk of breast and ovarian cancer in women and breast cancer in men. ${ }^{7,8}$ Population studies have shown a frequency of approximately $1.5 \%$ for this mutation in Ashkenazi Jews. ${ }^{6,9}$ Together, the $185 \mathrm{delAG}, 5382 \mathrm{insC}$, and $6174 \mathrm{del}$ T mutations account for

From the 'Department of Psychiatry and Behavioral Sciences; the 'Dcpartment of Molecular and Human Genetics; the ${ }^{3}$ Department of Medicine; the 'Department of Obstetrics and Gynecology; the ${ }^{5}$ Department of Surgery; the 'Department of Pediatrics; and the 'Texas Children's Cancer Center, Baylor College of Medicine. Houston, Texas.

Address correspondence to: Lois C. Friedman, PhD, Department of Psychiatry and Behavioral Sciences, Baylor College of Medicine, One Baylor Plaza, Houston, Texas 77030. E-mail: loisf@bcm.tmc.edu

(c) 1999 Genetics in Medicine a carrier frequency of approximately $2.7 \%$ in Ashkenazim. ${ }^{6,10}$

The discoveries of the BRCA1 and BRCA2 genes have provided the opportunity for DNA testing of at-risk individuals. However, the current status of molecular testing is such that the majority of people will receive negative test results. ${ }^{11}$ In a large collaborative study of 798 women at high risk for susceptibility mutations for breast and/or ovarian cancer, $B R C A 1$ mutations were identified in only $12.8 \%$ of the individuals tested. ${ }^{11}$ The problem is complicated further by the occurrence of probable false negatives, that is, when there appears, by history, to be a dominant gene in the family predisposing to breast cancer but DNA testing of an affected family member does not detect a mutation. In familial colon cancer testing, previous authors have identified up to $31.6 \%$ misinterpretation of false negative results as true negatives by physicians ordering the test. ${ }^{12}$ In addition to the possibility that medical providers might not have adequate knowledge to interpret DNA test results, negative test results might provide false reassurance to individuals who are at increased risk because of their family histories. These people may not perceive themselves to be at increased risk and may not be compliant with recommended surveillance. There also is the possibility that receiving a negative test result may be associated with "survivors' guilt." Individuals may feel guilty that they were given "good news" while other family members may be mutation carriers and/or have developed breast or ovarian cancer. Because of these reasons, it is important to determine the psychological responses of individuals enrolled in genetic testing programs who receive negative results. Studying the psychological consequences of DNA testing in Ashkenazim 
specifically is particularly relevant, given the recent discoveries of frequent mutations that predispose this population to breast and ovarian cancers.

The purpose of this study is to examine the psychological impact of DNA testing for a common mutation, 185delAG, in a community-based sample of Ashkenazi Jews. We examined here only those participants who received negative test results. This 6-month follow-up is part of an ongoing study of the psychological and behavioral impact of DNA testing for this mutation in Ashkenazim after notification of genetic test results. The main independent variable was risk status. The dependent variable, psychological distress, was assessed by a general measure and a cancer-specific measure at three time points: baseline and 1 and 6 months after DNA testing.

We hypothesized that those individuals who were at increased risk would report greater distress than those whose risk was average. We also hypothesized that distress levels would go up among the increased risk group and down among the average risk group after receiving test results. The role of demographic factors including gender, age, education, and marital status also was examined.

\section{METHODS}

\section{Subject selection}

The study sample was recruited from more than 50,000 Jewish individuals residing in Houston, Texas between April 9 and May 7, 1996. Announcements were placed in local Jewish publications and newsletters of Jewish organizations and were posted at local synagogues, the Jewish Community Center, and Baylor College of Medicine. Participants also were recruited through radio and television stations and local newspapers. Announcements described the study and indicated that participants would be offered educational sessions about the genetics of breast and ovarian cancer and surveillance and prevention options, asked to complete a series of questionnaires, offered DNA testing at no cost, and given their test results.

Study inclusion criteria included the following: (1) 21 years of age or older; (2) at least 50\% Ashkenazi (Central or Eastern European) Jewish ancestry; (3) ability to speak, read, and write English; and (4) ability to provide informed consent. Richards et al. ${ }^{13}$ provides a more detailed description of the subject selection.

\section{Procedures}

Procedures and consent forms were approved by the Baylor College of Medicine Institutional Review Board. Before the educational session, participants completed a questionnaire assessing knowledge of breast and ovarian cancer genetics. Sessions lasted approximately 2 hours and were limited to 50 participants each. Identical educational material (prewritten script) was presented by either one of two geneticists or a Master's level genetic counselor. The first half of the 45-minute slide presentation included information about the frequency of breast and ovarian cancer in the general population and in the Ashkenazi Jewish population; the discovery of the 185delAG mutation; and the associated risk of breast, ovarian, and colon cancer in women and colon and prostate cancer in men. The second half of the slide presentation focused on the goals of the study; eligibility criteria; possible outcomes of testing and implications for sur- veillance based upon personal and family histories of breast and ovarian cancer; American Cancer Society (ACS) recommended screening guidelines for breast, prostate, and colon cancer in the general population; and current surveillance and prevention guidelines for those at increased risk for breast and ovarian cancer. A discussion of the consent process, including potential risks and benefits of genetic testing, was outlined and discussed. Participants were told that there was a potential risk of insurance discrimination even if testing was performed as part of a study. Participants were informed that if they discussed their DNA test results with their physicians and obtained more frequent cancer surveillance or prophylactic surgeries, this information would be part of their medical record and would be available to insurance companies. It was stressed that participants did not have to agree to the genetic testing to participate in the study, and that we were just as interested in those who declined testing as those who chose to be tested. The decision to have or decline testing was reported privately after each session. A clinical psychologist was present at all of the sessions and participated in the question and answer period after each presentation.

Follow-up questionnaires were mailed approximately $\mathrm{l}$ and 6 months after notification of DNA results. The study protocol also includes a yearly follow-up for a 5-year period. For the first and second follow-ups, 309 individuals were mailed questionnaire packets. Participants who did not return the questionnaires received a second packet. A total of 227 participants (74\%) returned questionnaires at all three time points (baseline and 1 - and 6-month follow-ups). Of the 309 participants, 289 were tested for the $185 \mathrm{del} A \mathrm{G}$ mutation. The six participants who tested positive were excluded from the present study. Of the 283 mutation-negatives, 199 provided complete data on the measures used here.

\section{Risk status}

Participants were categorized as average risk if they tested negative and had negative family and personal histories of breast and ovarian cancer. Their risk of developing breast or ovarian cancer therefore was that of the general population. Participants were categorized as increased risk if they tested negative but had positive family or personal histories of breast or ovarian cancer, and either had no information about their affected relatives' genetic status or had affected relatives with negative DNA test results. A positive family history was defined as one first- or two second-degree relatives with breast cancer (age $<50$ years) or ovarian cancer.

\section{Notification of results}

Individuals who had negative DNA test results and no personal or family history of breast or ovarian cancer were notified by letter of their test results and told that their and their children's cancer risk remained the same as that of the general population. They also were provided with educational materials, including ACS cancer screening guidelines for breast and colon cancer in women and colon and prostate cancer in men.

Participants with negative DNA test results but positive family histories received letters notifying them of their test results, explaining that because of their positive family histories, their negative test results were not informative. They were given the 
option of clarifying their genetic risk by contacting their closest living relatives with breast or ovarian cancer to obtain consents and blood samples for DNA testing for 185delAG at no cost. They also were informed about the availability of individualized genetic consultation in the Houston area and were given educational materials, along with the recommendation that they follow ACS cancer screening guidelines. Participants with negative DNA test results but positive personal histories of breast or ovarian cancer also were contacted by mail and told that the test results did not clarify their genetic risk status either. A group conference with a medical oncologist, surgeon, and gynecologist was offered to discuss cancer screening. All participants who had negative DNA test results were given the opportunity to discuss their test results and concerns with a genetic counselor and a clinical psychologist. Further details concerning DNA testing procedures, molecular analysis for the $185 \mathrm{del} A \mathrm{AG}$ mutation, and notification of DNA testing results are provided elsewhere. ${ }^{13,14}$

\section{Measures}

Information regarding personal and family history of cancer and demographic characteristics was gathered, and number of psychological and behavioral (i.e., cancer surveillance) selfreport questionnaires were administered. Questionnaires were administered after participants were fully informed about the genetic testing protocol. In this paper, we report on the demographic and personal and family history information as well as the measures of psychological distress.

\section{Demographic variables}

Gender, age, educational level, and marital status were measured after the educational session.

\section{General psychological distress}

The Profile of Mood States-Short Form (POMS-SF), ${ }^{15}$ a brief version of the Profile of Mood States (POMS), ${ }^{16}$ is a 37 -item measure that yields scores for each of six subscales (TensionAnxiety; Anger-Hostility; Depression-Dejection; Vigor-Activity; Fatigue-Inertia; and Confusion-Bewilderment) as well as an overall Total Mood Disturbance score. In the current study, the Total Mood Disturbance score was used to assess general psychological distress experienced during the past week. Adequate reliability and validity for the POMS have been demonstrated. Cronbach's alpha (i.e., internal consistency) for this measure is 0.89 . The POMS-SF has been shown to be an excellent alternative to the POMS when a shorter version is desired. ${ }^{15}$ The POMS has been used in studies of female first-degree relatives of ovarian cancer patients ${ }^{17,18}$ and women with family histories of breast cancer receiving breast cancer risk counseling. ${ }^{19}$

\section{Cancer-specific distress}

The intrusion scale of the Impact of Events Scale (IES) ${ }^{20}$ consists of seven items used to assess intrusive thoughts and images, strong feelings, and dreams about a stressful event. In this study, the event was cancer. For example, participants were asked how often they thought about cancer when they did not mean to and indicated their response on a Likert scale ranging from 0 ("Not at All") to 5 ("Often"). Participants indicated how frequently the comments were true for them during the past 7 days. This measure has adequate reliability and validity. Cronbach's alpha is 0.89 . The IES has been used in other studies to measure psychological functioning of women at high risk for breast ${ }^{19,21}$ and ovarian $^{18}$ cancer and women tested for a BRCAl mutation. ${ }^{22,23}$

\section{Cancer history}

Personal history of cancer was obtained by asking participants to indicate whether they ever had been diagnosed with breast (bilateral or unilateral), ovarian, colon, prostate, or other cancer, and if so, their age at diagnosis. Family history of cancer was obtained by asking participants to complete a chart for first and second degree family members with breast, ovarian, colon, prostate, or other cancer, including age at diagnosis. Studies have shown excellent concordance between recall of breast cancer in family members and information gathered from medical records. ${ }^{24,25}$ In a recent validation study of reports of family cancer histories by probands and close relatives, results showed high reliability between self-reported familial cancer and data obtained from medical records and/or death certificates. ${ }^{26} \mathrm{Per}-$ centage agreement was particularly high for recall of breast cancers $(98.3 \%)$.

\section{Data analyses}

Data analyses were done in two stages. First, relationships between demographic variables (age, sex, years of education, and marital status) and measures of psychological distress (the POMS-SF and the IES) were examined to determine if these were related to one another, so that demographic variables related to measures of psychological distress could be included in any analyses. This was done using Pearson product-moment correlations. Gender and marital status were dummy coded $(0=$ male, $1=$ female; $0=$ not married, $1=$ married).

The second analysis was a logistic regression. The dichotomous outcome measure for this analysis was level of risk (average versus increased). Independent measures were the POMS-SF and the IES, which were assessed at three time points. The analysis was done hierarchically, with demographic measures entered in the first block, and the two measures of psychological distress gathered at three time points entered sequentially as three separate blocks. This was done to determine whether the two groups differed at baseline, after taking demographic differences into account, and so any group differences at the second and third time points would be adjusted both for these initial differences and for any demographic differences related to psychological distress. When applicable, all $P$ values were obtained from two-sided tests. A total of 199 individuals provided complete information on the measures included in the analysis.

\section{RESULTS}

\section{Participant characteristics}

Of the 333 individuals who attended the educational session, 309 (93\%) consented to participate in the study. Data are not available on the individuals who declined to participate. Almost all those who consented to participate $(94 \%)$ also requested DNA testing. Table 1 presents the demographic and cancer history characteristics of participants included in the present analyses. The majority were female $(89 \%)$, between the ages of 40 and 59 years $(66 \%)$, and married (76\%). Most were college educated $(98 \%)$, with $54 \%$ having a graduate education. Personal 
Table 1

Participant characteristics

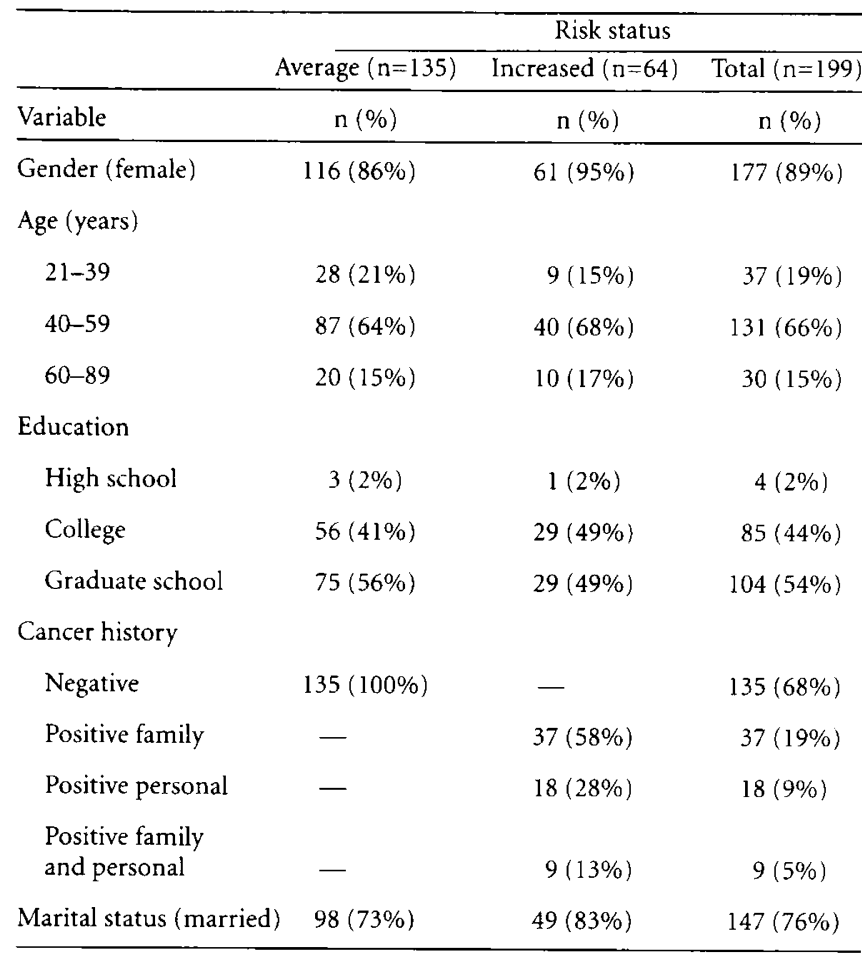

and family histories of breast or ovarian cancer were categorized as follows: negative family history (68\%); positive family history (19\%); positive personal history of breast or ovarian cancer (9\%); and both personal and family histories of breast or ovarian cancer (5\%). Participants' reports of baseline cancer surveillance behaviors during the preceding year can be found in Richards et al. ${ }^{13}$ Approximately $85 \%$ of women without personal histories of breast cancer reported having had a physician breast and pelvic examination in the past year. Women 40 years and older were more likely to have had an annual mammogram and breast examination than younger women. There were no differences between younger and older women in the frequency of annual pelvic examinations.

\section{Attrition}

Attrition analyses were conducted to determine how those who failed to return questionnaires or provide complete data differed from those who provided complete data on baseline measures. Both multivariate and univariate tests were done, because 36 of the participants who did not return questionnaires at both follow-ups also failed to provide complete baseline data on all variables. Multivariate analyses were limited to those 58 participants who did not return questionnaires at both follow-ups, but did provide complete baseline data. Logistic regression was used to compare the 199 participants included in the present study with these 58 participants. Variables included in this analysis were the POMS-SF, the IES, age, years of education, gender, risk status, and marital status.

The logistic regression indicates that the two groups differ significantly at the multivariate level $\left(\chi^{2}, 7 \mathrm{df}=16.6, P=0.02\right)$. The standardized regression coefficients for the variables in the equation indicate that the coefficient associated with marital status is significant $(P=0.02)$. Those who returned questionnaires at all three time points were more likely to be married.

\section{Relationships between demographics and psychological distress}

The analysis examining univariate relationships between gender, age, marital status, and POMS-SF (general distress) scores and IES (cancer-specific distress) scores indicate that gender was significantly related to the IES ( $r=0.18, P<0.01)$, with women having higher scores. Age was related negatively to the IES $(r=-0.14, P<0.05)$, with younger people scoring higher. Gender and age therefore were included in the hierarchical logistic regression.

\section{Comparison of increased risk and average risk groups on psychological distress}

Logistic regression analyses indicate that the groups differed significantly on the demographic measures (gender and age) entered in the first block $\left(\chi^{2}, 2 \mathrm{df}=11.3, P=0.003\right)$. The addition of the POMS-SF and IES baseline measures in the second block did not result in a significant change $\left(\chi^{2}, 2 \mathrm{df}=2.7, P=\right.$ 0.26 ); nor was the addition of the same measures at the 1-month follow-up (third block) associated with a significant change $\left(\chi^{2}\right.$, $2 \mathrm{df}=2.2, P=0.34$ ). The addition of the distress measures at the 6-month follow-up (fourth block) also was not associated with a significant change $\left(\chi^{2}, 2 \mathrm{df}=5.4, P=0.07\right)$.

With the demographic measures and the measures of psychological distress at baseline, and 1-month and 6-month follow-ups in the equation, the difference between the two groups was statistically significant $\left(\chi^{2}, 9 \mathrm{df}=21.6, P=0.006\right)$. Standardized regression coefficients for gender $(-1.80)$, age $(0.03)$, and the IES $(0.09)$ differed significantly from zero $(P$ values $<$ 0.05 ). Although the overall model was significant, the difference between the groups was based primarily upon differences in IES scores, age, and gender at the 6-month follow-up $(P=0.02, P$ $=0.03$, and $P=0.02$, respectively). The fact that the coefficient associated with the cancer-specific IES distress score at the 6month follow-up was significant indicates that differences on this measure account for variability between the two groups after adjusting for differences at baseline and 1 month and for demographic differences between the two groups.

Figure 1 graphically illustrates the means and standard deviations for the two groups on the IES at baseline, 1-month and 6-month follow-up. The wide variability in scores is taken into account by the logistic regression analysis. Decreases in cancerspecific psychological distress had occurred in both groups at the 1-month follow-up. At the 6-month follow-up, the level of distress in the average risk group had decreased even more, whereas that in the increased risk group had begun a return to baseline. Compared with a mean distress score of $21.4 \pm \beta 8.6$ obtained by patients treated at a stress disorders clinic, ${ }^{20}$ however, baseline, 1-month, and 6-month scores of both the average and increased risk groups were all relatively low (range of means $=2.9 \pm 4.7$ to $6.3 \pm 7.1$ ).

\section{DISCUSSION}

The present study examined the psychological impact of receiving a negative $B R C A 1$ mutation test result in Ashkenazim. After controlling for gender and age, increased risk participants 


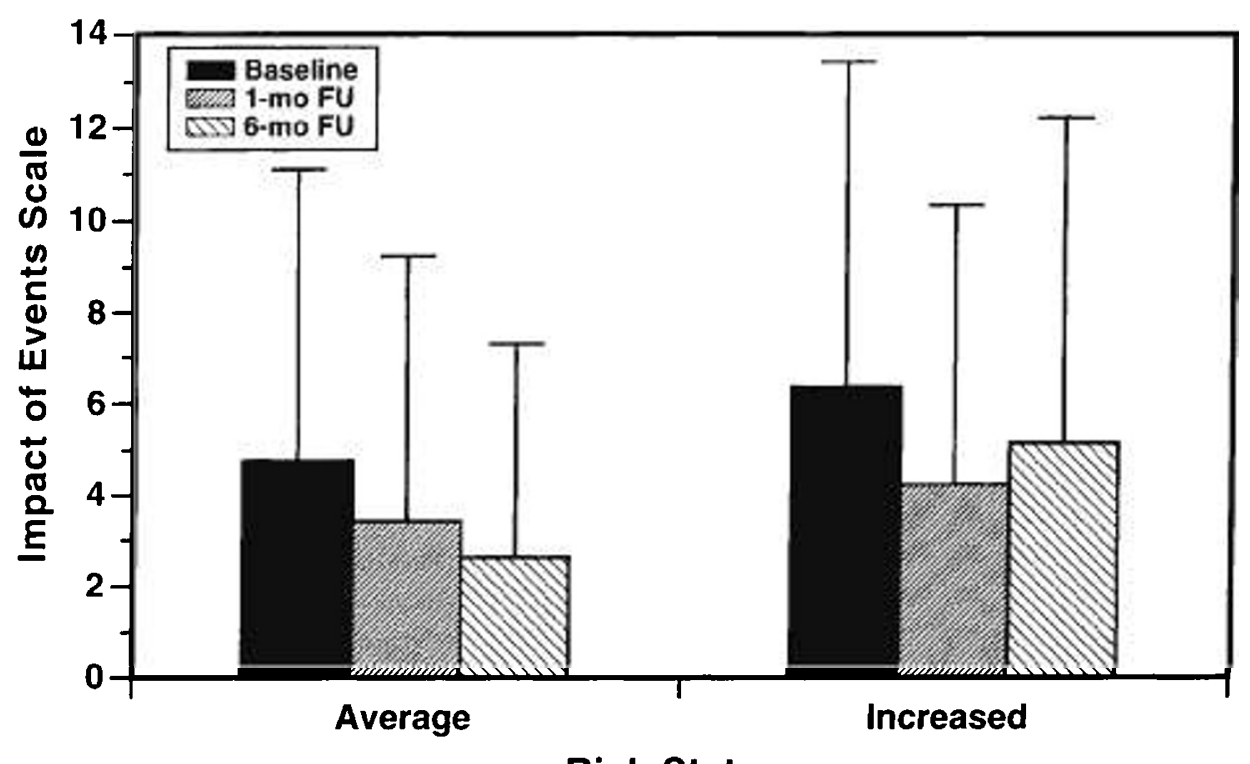

Risk Status

Fig. 1 Mcans and standard deviations fior measures of cancer-specific distress (Impact of Events Scale) for average risk and increased risk participants at baseline, 1-month, and 6-month follow-up.

reported slightly but significantly higher levels of cancerspecific distress than average risk participants at the 6-month follow-up. For all participants, general psychological distress declined during this 6-month period. Cancer-specific distress had declined among all participants at the 1-month follow-up. While the average risk group's cancer-specific distress level continued to decline 6 months after notification of DNA testing results, the increased risk group's distress level had begun a climb back to baseline. It is important to keep in mind, however, that although differences in the cancer-specific distress levels obtained by our sample, both at baseline and at the two follow-ups, were statistically significant, none of the absolute levels observed reflected especially high degrees of distress.

For those participants with personal and/or family histories of breast or ovarian cancer (the increased risk group) who may have anticipated being mutation carriers, negative test results could have been received with relief. Some of the psychological benefits experienced by these participants 1 month after testing, however, were lost by the 6-month follow-up. Because we informed the increased risk participants that their positive family histories made their negative test results uninformative, their elevated distress (compared with the average risk group) was appropriate. These individuals need to be more concerned about their cancer risk because of their positive family histories. It will be important, in our long-term follow-up, to determine not only whether the increased risk group continues to manifest elevated concerns about cancer, but also whether these concerns affect female participants' compliance with cancer surveillance recommendations.

The data suggest that women and younger people may need more counseling during genetic testing. Our findings are consistent with those of a recent study of patients with colorectal cancer participating in a DNA testing program for hereditary nonpolyposis colon cancer, in which psychological distress was found to be associated with being female, being younger, and not having adequate social support. ${ }^{27}$

There are several methodological issues which need to be addressed. Because our participants were highly educated individuals of Ashkenazi Jewish ancestry, the generalizability of the results needs to be considered. Our participants were very interested in genetic testing despite its potential risks and limitations, which included the possibility of insurance and employment discrimination and limited knowledge about the effectiveness of existing surveillance and prevention options. Jewish focus groups have expressed continued interest in DNA testing for breast and ovarian cancer despite being informed of the risks and limitations associated with testing. ${ }^{28}$ In contrast, non-Jewish focus groups' interest in testing decreased once they had been educated about these issues. ${ }^{28}$ It is unclear what the psychological consequences after receipt of negative DNA test results would be for a less educated and/or non-Jewish population.

Furthermore, all of the participants attended an educational program before genetic testing and had a genetic counselor and clinical psychologist available to address any questions or concerns both before and after testing. Psychological distress levels might be higher than in a comparable group undergoing DNA testing in a clinical setting that does not provide similar services.

It also is possible that participants' baseline levels of cancerspecific distress would have been lower if the assessments were done before the educational program and consent process. The 6-month distress levels reported by the increased risk group may actually be above their preeducation baseline levels, thus underestimating the distress experienced by this group. However, as mentioned earlier, distress levels obtained at baseline, 1-month, and 6-month follow-ups were all relatively low.

Results from this community-based genetic testing program suggest that genetic testing conducted in a research setting is not necessarily associated with a deleterious psychological 
impact on individuals who receive negative test results even when the results are uninformative. General psychological distress declined among both groups after DNA testing. The increased risk group's cancer-specific distress level at the 6month follow-up, although lower than at baseline, was significantly greater than that of the average risk group. Further research is needed to replicate these findings and to examine the role of expectations in psychological adjustment after genetic testing. The rapid rate of discovery of genetic mutations which increase an individual's susceptibility to developing cancer highlights the need to understand factors associated with both positive and negative psychological and behavioral consequences of genetic testing. Given the continuing controversies about mutation detection for $B R C A 1$ and $B R C A 2$, additional data with regard to the impact of both positive and negative test results are important.

\section{Acknowledgments}

Supported in part by the Jewish Institute of Medical Research Endowment Fund and the JIMR/Glesby Endowment Fund of the Endowment Fund of the Jewish Community of Houston.

\section{References}

1. American Cancer Society. Cancer facts and figures-1997. Atlanta: American Cancer Society, 1997

2. Claus EB, Risch N, Thompson WD. Genetic analysis of breast cancer in the Cancer and Steroid Hormone Study. Am J Hum Genet 1991:48:232-242.

3. King MC, Rowell SE, Love SM. Inherited breast and ovarian cancer: What are the risks? What are the choices? JAMA 1993;269:1975-1980.

4. Couch FJ, DeShano ML, Blackwood MA, Calzone K, Stopfer J, Campeau L, Ganguly A, Rebbeck T, Weber BL. BRCAI mutations in women attending clinics that evaluate the risk of breast cancer. N Engl J Med 1997;336:1409-1415.

5. Struewing IP, Hartge P, Wacholder S, Baker SM, Berlin M, McAdams M, Timmerman MM, Brody LC, Tucker MA. The risk of cancer associated with specific mutations of BRCA I and BRCA2 among Ashkenazi Jews. N Engl J Med 1997;336:1401-1408.

6. Roa BB, Boyd AA, Volcik K, Richards CS. Ashkenazi Jewish population frequencies for common mutations in BRCA1 and BRCA2. Nat Genet 1996;14:185-187.

7. Couch FJ, Farid LM, DeShano ML, Tavtigian SV, Calzone K, Campeau L, Peng Y, Bogden B, Chen Q, Neuhausen S, Shattuck-Eidens D, Godwin AK, Daly M, Radford DM, Sedlacek S, Rommens J, Simard J, Garber J, Merajver S, Weber BL. BRCA2 germline mutations in male breast cancer cases and breast cancer families. Nat Genet 1996;13:123-125.

8. Neuhausen S, Gilewski T, Norton L, Tran T, McGuire P, Swensen J, Hampel H, Borgen P, Brown K, Skolnick M, Shattuck-Eidens D, Jhanwar S, Goldgar D, Offit K. Recurrent $B R C A 26174 \mathrm{delT}$ mutations in Ashkenazi Jewish women affected by breast cancer. Nat Genet 1996;13:126-128.
9. Oddoux C, Struewing IP, Clayton CM, Neuhausen S, Brody LC, Kaback M, Haas B, Norton L, Borgen $P$, Jhanwar $S$, Goldgar D, Ostrer $H$, Offit $K$. The carrier frequency of the BRCA2 6174delT mutation among Ashkenazi Jewish individuals is approximately $1 \%$. Nat Genet 1996;14:188-190.

10. Ford D), Easton DF, Petro J. Estimates of the gene frequency of $B R C A /$ and its con tribution to breast and ovarian cancer incidence. Am J Hum Genet 1995;57:1 457-1462.

11. Shattuck-Eidens D, Oliphant A, McClure M, McBride C, Gupte J, Rubano T, Pruss D, Tavtigian SV, Teng DH, Adey N, Staebell M, Gumpper K, Lundstrom R, Hulick M, Kelly $M$, Holmen J, Lingenfelter B, Manley S, Fujimura F, Luce M, Ward B, CannonAlbright $\mathrm{L}$, Stecle $\mathrm{L}$, Offit $\mathrm{K}$, Thomas A. BRCAl sequence analysis in women at high risk for susceptibility mutations. JAMA 1997:278:1242-1250.

12. Giardiello FM, Brensinger JD, Petersen GM, Luce MC, Hylind IM, Bacon JA, Booker SV, Parker RD, Hamilton SR. The use and interpretation of commercial APC gene Iesting for familial adenomatous polyposis. N Engl J Med 1997;336:823-827.

13. Richards CS, Ward PA, Roa BB, Friedman LC, Boyd AA, Kuenzli G, Dunn JK, Plon SE. Screening for 185dcLAG in the Ashkenazim. Am J Hum Genet 1997;60:1085-1098

14. DeMarchi IM, Richards CS, Fenwick RG, Pace R, Bcaudet AL. A robotics-assisted procedure for large scale cystic fibrosis mutation analysis. Hum Mutat 1994;4:281-290.

15. Curran SL, Andrykowski MA, Studts JL. Short form of the Profile of Mood States (POMS-SF): Psychometric information. Psychol Assessment 1995;7:80-83.

16. MC Nair D, Lorr M, Droppleman L. Profile of Mood States. San Diego, California: EdITS/Educational and Industrial Testing Service, 1981

17. Lerman C, Daly M, Masny A, Balshem A. Attitudes abut genetic testing for breastovarian cancer susceptibility. J Clin Oncol 1994;12:843-850.

18. Schwartz MD, Lerman C, Miller SM, Daly M, Masny A. Coping disposition, perceived risk and psychological distress among women at increased risk for ovarian cancer. Health Psychol 1995;14:232-253.

19. Lerman C, Schwartz MD, Miller SM, Daly M, Sands C, Rimer BK. A randomized trial of breast cancer risk counseling: Interacting effects of counseling, educational level, and coping style. Health Psychol 1996;15:75-83.

20. Horowitz M, Wilner N, Alvarez W. Impact of Events Scale: A measure of subjective stress. Psychosom Med 1979;41:209-218.

21. Kash KM, Holland IC, Halper MS, Miller DG. Psychological distress and surveillance behaviors of women with a family history of breast cancer. I Natl Cancer Inst 1992;84:24-30.

22. Croyle RT, Smith KR, Botkin IR, Baty B, Nash I. Psychological responses to BRCAl mutation testing: Preliminary findings. Health Psychol 1997;16:63-72.

23. Lerman C, Schwartz MD, Lin TH, Hughes C, Narod S, Lynch HT. The influence of psychological distress on use of genetic testing for cancer risk. J Consult Clin Psychol 1997;65:414-420.

24. Love RR, Evans AM, Josten DM. The accuracy of patient reports of a family history of cancer. J Chronic Dis 1985;38:289-293.

25. Parent M-E, Ghadirian P, Lacroix A, Perret $\mathrm{C}$. The reliability of recollections of family history: Implications for the medical provider. J Cancer Edtc 1997;12:114-120.

26. Airewele G, Adatto P, Cunningham J, Mastromarino C, Spencer C, Sharp M, Sigurdson A, Bondy M. Family history of cancer in patients with glioma: A validation study of accuracy. I Natl Cancer Inst 1998;90:543-544.

27. Vernon SW, Gritz ER, Peterson SK, Amos CI, Perz CA, Baile WF, Lynch PM. Correlates of psychologic distress in colorectal cancer patients undergoing genetic testing for hereditary colon cancer. Health Psychol 1997;16:73-86.

28. Geller G, Bernhardt BA, Helzlsouer $K$, Holtzman N, Stefanek M. Wilsox PM. Informed consent and BRCA1 testing. Nat Ginet 1995;11:364. 